\title{
INVEESTIGACIONES GEOGRAFICAS
}

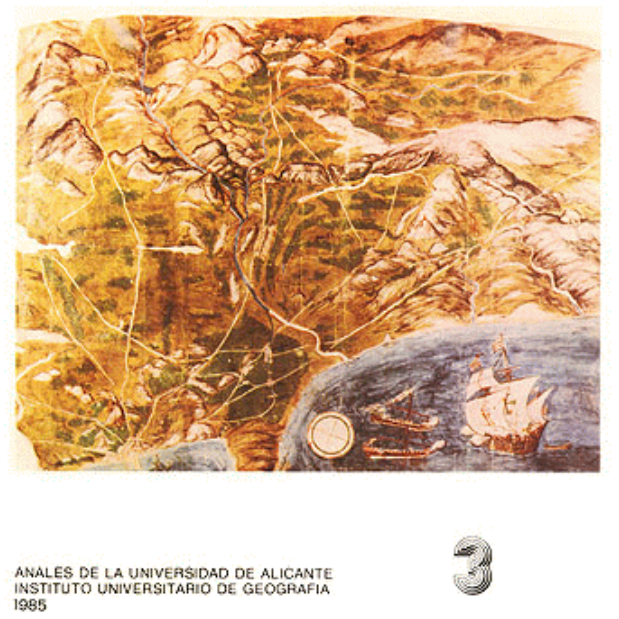

[7]

APLICACIÓN DE LA FOTOGRAFÍA AÉREA EN LA CARTOGRAFÍA DE SUELOS

\section{Enrique Matarredona Coll}

Un estudio cartográfico, en edafología, consiste en el estudio de los suelos enmarcados en el paisaje, sin disociarles de los restantes elementos del medio. Se trata, pues, de una aproximación global y sintética, eminentemente geográfica, que permita comprender las relaciones existentes entre las diversas unidades de suelos.

Con el presente estudio se pretende ofrecer un método de trabajo empleado en cartografía de suelos basado eminentemente en técnicas de fotointerpretación. Con ese objetivo -como metaestructuramos el trabajo en tres apartados: en primer lugar, abordamos, de una forma esquemática, la situación de la cartografía de suelos en nuestro país; seguidamente, desarrollamos el método propuesto, que ya hemos aplicado en tres comarcas alicantinas; y finalmente, hacemos referencia a la aplicación práctica de esta metodología a un área piloto de la provincia de Alicante: zona abarcada por el fotograma $\mathrm{n}^{\mathbf{0}} 7243$ del vuelo de 1956.

Como quiera que el objetivo es fundamentalmente metodológico, en este último punto -aplicación del sistema a un área concreta- hemos prescindido de análisis granulométricos y determinaciones físico-químicas que, obviamente, se hacen necesarios y aconsejables en los estudios de cartografía de suelos como ponemos de manifiesto en otros trabajos (vid. notas núms. $8,9$ y 10$)$.

\section{APROXIMACIÓN A LA SITUACIÓN DE LA CARTOGRAFÍA DE SUELOS}

La cartografía de suelos tiene por objeto delimitar zonas [8] homogéneas de suelos. SIMONSON (1952) definió la cartografía de suelos como el proceso de inventariar dicho recurso en una zona determinada, incluyendo las características edáficas más importantes, la clasificación 
de los suelos en categorías establecidas sistemáticamente y la localización y trazado de sus límites sobre mapas. ${ }^{1}$

La unidad de estudio de dicho proceso cartográfico, el individuo suelo, es resultado de la combinación de los cinco factores formadores: material original, clima, potencial biótico, fisiografía y tiempo (JENNY, 1945). Sin embargo, debido a la complejidad de las formaciones edáficas, un individuo-suelo, representado por una unidad cartográfica de un mapa detallado de suelos (POWELL y SPRINGER, 1965; WILDING, 1965) puede incluir otros individuos-suelos distintos (BUOL, 1973). Por ello se suelen utilizar las asociaciones cartográficas de suelos, entendidas como la unidad de dos a más suelos que podrían ser separados en reconocimientos de detalle (E 1:20.000 o mayores).

Dada la enorme extensión y variabilidad del material de estudio, el desarrollo progresivo de la investigación en el campo de la Cartografía de suelos precisa del inventario continuado en las distintas zonas geográficas. Por ello, paralelo al quehacer puramente científico, debe existir un reconocimiento sistemático que haga aplicación en cada momento de los conocimientos y técnicas proporcionados por la investigación.

En España, la cartografía sistemática pertenece, en la mayoría de los casos, al tipo generalizado, según se desprende del análisis de trabajos realizados, entre otros, por el CEBAC relativos a estudios agrabiológicos de las provincias de Sevilla (1962), Cádiz (1963) y Córdoba (1972); por el CEBAS referente a un estudio similar sobre la provincia de Murcia (1968); por el Instituto Nacional de Edafología y Agrobiología, con el mapa de suelos E 1/250.000 de Guadalajara (1970); por el Centro de Edafología y Biología Aplicada de Salamanca, con un mapa de suelos de la provincia de Cáceres (1972), estudios, todos ellos, a nivel de inventario de suelos que se consideran de limitada utilidad ${ }^{2}$.

Asimismo, en este punto señalamos que en 1956 se estableció [9] un acuerdo entre el Instituto Nacional de Edafología (CSIC) y el Mapa Agronómico Nacional (Ministerio de Agricultura), por el cual se realizarían mapas a nivel de unidades de aplicación, es decir «series de suelos». Sin embargo, la utilización de una escala inadecuada -puesta de manifiesto en los Mapas provinciales de suelos E 1/200.000 de Cádiz (1971) y Sevilla (1975) del Instituto Nacional de Investigaciones Agrarias- y la extinción gradual de las actividades de dicho Mapa han determinado la falta, casi total de reconocimientos detallados de suelos en España ${ }^{3}$.

Entre los pocos ejemplos de mapas detallados se podrían citar algunos trabajos de centros dependientes del CSIC, motivo de publicaciones internas; de la Diputación Foral de Navarra, con fines catastrales y experimentales; y del IRYDA, para estudios de riego y drenaje. Este Instituto ha llevado a cabo también una cartografía de suelos relativamente extensa, a nivel de semidetalle, y en colaboración con el Centro de Estudios Hidrográficos y Confederaciones Hidrográficas.

Otros tipos especiales de estudios cartográficos de suelos son los desarrollados por DEL VILLAR (1937), relativo a los «suelos de la Península Luso-Ibérica»; los del Ministerio de Agricultura (1958) sobre los grupos principales de suelos de la España Peninsular (E 1/1.300.000); y los del Instituto Nacional de Edafología y Agrobiología (1968) referente al Mapa de suelos de España (E 1/1.000.000) dirigido por GUERRA DELGADO ${ }^{4}$.

Aparte de los citados, que tienen un carácter edafológico más notable, señalamos, entre otros, una serie de mapas de suelos, simples esquemas en algunos casos, que aparecen con el carácter de mapas temáticos dentro de conjuntos cartográficos regionales o provinciales, tales como el Mapa de suelos de Canarias (1/400.000) con terminología de la FAO-UNESCO; el Mapa de Sols de Baleares (1/1.000.000) con taxonomía de Kubiena, cuya elaboración corrió a cargo del Departamento de Geografía de Palma de Mallorca; el Mapa de Sols de Catalunya (1/2.000.000), también con taxonomía de Kubiena, realizado por el equipo que dirige M. ${ }^{\mathrm{a}}$ de BOLOS y el Mapa de suelos de Navarra (1/900.000), con nomenclatura de FAO. ${ }^{5}$ [10]

En lo que se refiere al País Valenciano, muy pocos son los estudios publicados hasta la 
fecha sobre suelos levantinos que tengan un cierto aprovechamiento para estudios geográficos. Los trabajos que se realizan sobre Edafología están controlados, bien por departamentos universitarios o por centros específicos vinculados al CSIC, como el CEBAS de Murcia que extiende su radio de acción por ámbitos alicantinos; en cualquier caso, suele tratarse de estudios muy específicos: análisis de suelos que buscan la vertiente aplicada a la agricultura ${ }^{6}$.

Así, en la Escuela de Ingenieros Agrónomos de Valencia se han realizado una serie de trabajos para el IRYDA sobre terrenos con proyectos de nuevos regadíos. Más próximos a la disciplina geográfica se sitúan los estudios del Departamento de Edafología de la Facultad de Farmacia, que trabaja sobre suelos de todo tipo (cultivados y no cultivados), estableciendo conexiones entre geomorfología y suelos?

Asimismo, la línea de investigación del CEBAS (Murcia) se manifiesta, en lo que concierne a la provincia de Alicante, en los trabajos de ALEIXANDRE y PINILLA (1968), CARPENA (1969, 1971, 1972), SÁNCHEZ (1973, 1977), CANALES (1972), entre otros, en cualquier caso, el tratamiento de todos ellos es muy específico y tienen poco contacto con el campo geográfico, excepción hecha del Estudio agrobiológico de Orihuela-Dolores o el capítulo de Cartografía de suelos de la tesis doctoral de CANALES.

En lo que se refiere a la provincia de Castellón, debemos citar la tesis doctoral de JIMÉNEZ BALLESTA (1976), así como trabajos posteriores realizados por el citado investigador en colaboración con GUERRA DELGADO (1980) sobre suelos castellonenses.

Los trabajos esencialmente cartográficos son todavía más escasos: el Mapa de suelos de la provincia de Valencia (1939) E 1/200.000, resulta claramente anacrónico; lo mismo que el realizado en la provincia de Alicante a la misma escala. También ha quedado desfasado el Mapa Agronómico Nacional (1952), del que en el País Valenciano sólo fue realizada la Hoja 747 correspondiente a Sueca (Valencia). [11]

Mejor tratamiento tienen los mapas de Asociaciones y Capacidad de Uso del suelo de la provincia de Castellón de JIMÉNEZ BALLESTA, que se han elaborado siguiendo los métodos y procesos propuestos por el Soil Conservation Service (USA) y las directrices del Departamento de Suelos del Instituto de Edafología y Biología Vegetal del CSIC y del Servicio de Reconocimiento y Ordenamiento Agrario de Portugal, así como el apéndice cartográfico sobre Capacidad de Uso agrícola de los suelos del País Valenciano, publicado en 1982 (E 1/100.000) por PREVASA y orientado hacia la ordenación del territorio de la Comunidad Valenciana.

\section{UN MÉTODO DE TRABAJO EN LA CARTOGRAFÍA DE SUELOS}

En el presente estudio vamos a hacer referencia a un método de trabajo, en Cartografía de suelos, que hemos aplicado en dos comarcas alicantinas: el Alto Vinalopó ${ }^{8}$ y el Campo de Alicante $^{9}$, así como en el Bajo Vinalopó ${ }^{10}$. Con ello pretendemos exponer una normativa de estudio de los suelos, sobre todo desde el punto de vista cartográfico.

No es esta una faceta muy corriente en la ciencia del suelo, como hemos comentado anteriormente; para encontrar en nuestro país antecedentes de cartografía de suelos, ha sido necesario remontarse a los trabajos de HUGUET DEL VILLAR (1937), TAMES (1957), KLINGE (1957), ONTAÑON (1966), GUERRA (1968) o trabajos de escalas mayores realizados por el CEBAS de Murcia o el CEBAC de Sevilla, entre otros.

Pretendemos, como objetivo general, aportar un mayor conocimiento sobre la evolución y distribución de los suelos. A tal efecto, planteamos un método de trabajo que nos permita comprender mejor las leyes que rigen la distribución de los suelos y evaluar las posibilidades de empleo de las principales asociaciones cartográficas y su capacidad de uso.

A grandes rasgos, la metodología que proponemos incluye las siguientes fases:

\subsection{Fase PRE-CARTOGRÁFICA.}


Cuando se va a cartografiar una comarca o región, es [12] preciso, de entrada, buscar todas las fuentes de información: mapas topográficos y geológicos, estudios geográficos ya publicados, fotografías aéreas, etc.

En este punto, es necesario resaltar que la fotografía aérea constituye uno de los elementos esenciales. En los últimos años se ha generalizado la utilización de fotografías aéreas como documentos de base para la identificación y cartografía de fenómenos geográficos ${ }^{11}$.

La fotografía aérea muestra el aspecto de la superficie terrestre: formaciones vegetales, espacios cultivados, áreas desérticas, etc.; las diferencias de tinte de los fotogramas resultan no sólo del color del suelo y de su cobertura sino de la absorción y reflexión de la luz, incluso del grado de humedad que hace variar sensiblemente estas propiedades.

Asimismo, dentro de esta fase, se procede, en base a los objetivos perseguidos, a la selección de la escala de trabajo más adecuada. En el Alto Vinalopó, por ejemplo, dado que el estudio se realizó como un capítulo dentro de un análisis geográfico comarcal, se consideró como escala más apropiada la 1/50.000 -empleada en las distintas facetas del estudio general-; escala que, a nuestro juicio nos permitía comprender las leyes de distribución de los suelos, al tiempo que posibilitaba la cartografía de las principales asociaciones edáficas y su posterior comparación con otros mapas temáticos.

En lo que respecta al Campo de Alicante y al Bajo Vinalopó, se empleó como escala de prospección la 1/50.000, mientras que quedaba como escala de publicación la 1/100.000, recomendada en este tipo de trabajos por el Groupe d'Etudes des Problemes de Pedologie Appliquèe.

Por último, una vez elegida la escala, se hace necesario seleccionar la clasificación taxonómica más apropiada. En este punto optamos, sin abandonar la tradicional clasificación española de Kubiena, por la propuesta por el sistema U.S.D.A. en su publicación Soil Taxonomy «A Basic System of Soil Surveys», conocida como la 10a Aproximación americana.

En función de todo lo expuesto, los documentos empleados [13] fueron: los mapas topográficos del Servicio Geográfico del Ejército (1/50.000 y 1/100.000), los mapas geológicos publicados por el Instituto Geológico y Minero de España (1/50.000 y 1/200.000), así como los fotogramas (1/33.000), publicados por el Ejército del Aire, correspondiente al llamado «vuelo americano» (1956).

\subsection{PROSPECCIÓN DE RECONOCIMIENTO}

Esta fase tiene como objetivo el reconocimiento general del campo para familiarizarse con el área objeto de estudio. A tal fin se elaboran unos esquemas geológicos y geomorfológicos, así como, se puede llegar a la consideración de los factores climáticos que pueden influir en la repartición de los suelos.

También en esta fase se pueden observar cortes naturales, analizar la presencia de costras calcáreas y eflorescencias salinas, constatar la existencia y tipología de la cobertura vegetal, notar la delimitación de áreas de cultivo, etc.; observaciones y análisis que son de notable ayuda para la labor de fotointerpretación a realizar con posterioridad.

\subsection{Fase de FOTOINTERPRETACIÓN}

Se trata del momento clave dentro del método propuesto, toda vez que esta técnica -fotointerpretación- permite establecer ciertas relaciones entre algunos fenómenos, perfectamente representados sobre el fotograma (topografía, formas de modelado, cobertura vegetal, etc.), con las principales unidades del paisaje.

Por otro lado, el ámbito investigado, dominio mediterráneo nato, es lugar idóneo para esta aplicación, ya que este ambiente rexistásico facilita la correspondencia entre los grandes conjuntos paisajísticos y los grandes patrones de distribución de suelos. 
La delimitación de estos patrones edáficos dentro de los grandes conjuntos naturales se puede realizar mediante la búsqueda de áreas homogéneas, las cuales pueden clasificarse por su grado de parecido o diferencia. Este método fisiográfico, de aplicación universal, permite [14] identificar límites relacionados con las diferencias en procesos fisiográficos.

Se pueden marcar estas áreas bien por un símbolo (letras, cifras, signos, etc.), o bien señalando un límite: trazar límites presenta la ventaja de poder transcribirlos sobre otro documento, aparte del fotograma; por ejemplo, un mapa topográfico. Todo límite trazado sobre la fotografía aérea, según el principio precedente, corresponde a una realidad, en la medida que se le dan definiciones precisas de las zonas homogéneas marcadas y que todos los puntos del fotograma pertenecen a una de las zonas descritas.

Pero esto no significa que todas las zonas delimitadas correspondan a la realidad que se estudia. En efecto, la estructura que se establece puede ser más o menos variada. El inconveniente del trazado de límites temporales sobre la fotografía es el influenciar el trazado definitivo sobre el terreno. Por ello, se hace necesario, a veces, modificar en mayor o menor medida los límites temporales fotointerpretados después del estudio sobre el terreno, o se necesita a menudo borrar o modificar límites que parecen netos sobre el fotograma o, incluso, ajustar otros que no aparecen en ellos.

Se puede evitar este inconveniente por el empleo de símbolos; para ello se determinan en la fotografía las zonas homogéneas atendiendo, no solo a la tipología del roquedo, sino también a la combinación de una serie de determinantes directos del fotograma (tonalidad, textura, por ejemplo) e indirectos del entorno (constitución geológica, aspecto topográfico, tipo de cobertura vegetal, aprovechamiento del suelo, etc.), toda vez que las diferencias de tonalidad de las manchas sobre el fotograma pueden ser motivadas por la distinta coloración del suelo, provocada bien por la propia tipología del roquedo, bien por la presencia de elementos pedregosos, o por el distinto grado de humedad e incluso de cobertura vegetal. ${ }^{12}$

Así, tomando en consideración la combinación de esos criterios determinantes se pueden distinguir un cierto número de zonas homogéneas que se pueden representar en [15] el fotograma con un símbolo formado por los indicadores que se citan en el cuadro correspondiente.

Se clasifican las zonas homogéneas comenzando por las que ofrecen más contrastes y terminando por las que constituyen situaciones intermedias entre ellas. Cada zona diferente es marcada con un símbolo que servirá de guía para la delimitación definitiva, una vez realizada la fase de verificación sobre el terreno.

En cualquier caso, la búsqueda de estas áreas homogéneas se efectúa a través del estudio de ciertos elementos individuales considerados de importancia en edafología, tales como: criterios litológicos, formas individuales del paisaje, unidades geomorfológicos de base, pendientes, red hidrográfica, detalles visibles del suelo (encostramientos, humedad, variaciones de color, salinización), elementos relacionados con la cubierta vegetal, empleo agrícola del suelo, etc.

Una vez diferenciadas estas áreas homogéneas, se acoplan en fotomosaicos y después, con un pantógrafo óptico, se reducen a la escala de prospección (1/50.000) sobre el correspondiente mapa topográfico.

\subsection{PROSPECCIÓN DE VERIFICACIÓN}

Una vez concluido el período anterior -diferenciación de áreas homogéneas-, es preciso preparar el estudio del terreno. Cada zona homogénea debe ser analizada con detalle y estudiada, al menos, por un perfil. La fotografía permitirá elegir el emplazamiento, de forma que este perfil pueda ser considerado como «perfil modal».

En esta fase de trabajo de campo debe utilizarse aún la fotografía aérea, puesto que ésta muestra detalles que no aparecen en los mapas topográficos (por ejemplo, el parcelario) y que 
permiten precisar de forma más ajustada el emplazamiento de los perfiles y los límites discernidos sobre el terreno. La fotografía sirve entonces tanto de documento de orientación como de referencia.

El trabajo de campo con la fotografía permite también controlar la validez de las hipótesis hechas, permitiendo [16] establecer correlaciones entre la realidad pedológica y la imagen fotográfica.

Ciertos límites pueden ser trazados. Es preciso entonces detectar los datos del terreno (in situ) que permiten seguir estos límites sobre el fotograma. Es preciso, asimismo, verificar si las zonas homogéneas, precedentemente descritas en la fase de fotointerpretación, corresponden o no a las entidades pedológicas.

Desde el punto de vista de la fotointerpretación, esta fase parecería no aportar más que los elementos necesarios para el establecimiento de correlaciones terreno-fotografía. De hecho el trabajo de campo representa una fase esencial de la fotointerpretación: permite verificar si el conjunto pre-establecido es o no coherente con la realidad.

La confrontación visual y razonada entre el paisaje imaginario y el paisaje real permite, a menudo precisar y encontrar nuevas relaciones. Esta relación es una de las bases de la fotointerpretación pedológica y constituye la aportación principal de esta última a la cartografía de suelos.

En resumen, podemos decir que, en función de la información obtenida en las fases anteriores, se procede a la selección de «áreas de muestreo» que son verificadas en el campo mediante un número ordenado y adecuado de observaciones; áreas que se pueden relacionar con otras semejantes y que, en algún caso, pueden extrapolarse.

\subsection{Elaboración del MAPA DE ASOCIACIONES CARTOGRÁFICAS}

Paralelamente a la prospección de verificación se va confeccionando un inventario de unidades taxonómicas, se estudian sus asociaciones y se definen los límites entre ellas.

En este punto cabe indicar que, debido a los condicionamientos climáticos de tipología mediterránea, los procesos edáficos se realizan en estos entornos geográficos lenta e irregularmente; condicionamientos que impiden la formación de perfiles marcados y profundos, salvo en el [17] 


\section{CUADRO I}

METODOLOGIA DE TRABNO EN CARTOGRAFIA DE SUELOS

\begin{tabular}{|c|c|c|c|}
\hline Fase & Noebre & objetivo & Desarrollo \\
\hline 1 & Pre-cartogrsfica & Bosqueda fuentes de inforaseión & $\begin{array}{l}\text { Recopilación mapas topogrsficos y geolbzicos } \\
\text { sleceión de la escala de prospección } \\
\text { Referencia a la escala de publieación } \\
\text { Deterninación de la clasificación taxonomica }\end{array}$ \\
\hline ti & Reconociniento & $\begin{array}{l}\text { Conocintento del farea objeto de } \\
\text { estudio }\end{array}$ & 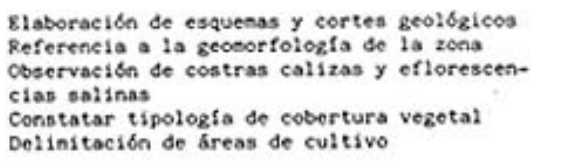 \\
\hline ItI & Fotointerpretacion & $\begin{array}{l}\text { Identificacidn de ifnites en zo- } \\
\text { nas hoeogtneas }\end{array}$ & 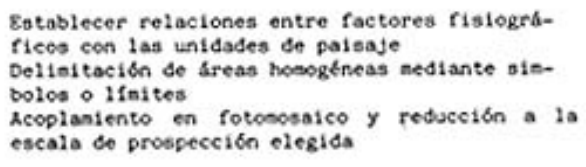 \\
\hline IV & Verificación & $\begin{array}{l}\text { Controlar la validez de las hi- } \\
\text { potesto realizidas }\end{array}$ & 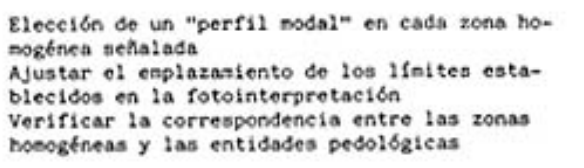 \\
\hline $\mathrm{v}$ & Cartografica & $\begin{array}{l}\text { Elaboracion del mapa de ascia- } \\
\text { ciones cartogríficas de suelos }\end{array}$ & $\begin{array}{l}\text { Confeccion de inventario do unidades taxcolai- } \\
\text { cas y asociacionea } \\
\text { Relacionar eatas asociaciones con las zonas } \\
\text { homogéneas delisitadas } \\
\text { Elaboración do la leyenda cartogrbfica sehalanm } \\
\text { do en cada asociación sus unidades taxonbnican }\end{array}$ \\
\hline
\end{tabular}

[18]

fondo de las depresiones. Por ello, los suelos se diferencian poco de la litología subyacente en cuanto a color y estructura, circunstancia que ayuda a la hora de cartografiar.

Por otro lado, el empleo de escalas del tipo 1/50.000 ó 1/100.000 hace imposible que hablemos de tipos de suelos, como hemos indicado anteriormente, toda vez que estos se presentan en manchas donde la separación entre ellas se hace difícil a esta escala de trabajo. Es por ello por lo que aconsejarnos la utilización de las asociaciones cartográficas, como unidades que sintetizan una determinada gama de suelos.

De cualquier modo, ultimados los esquemas de prospección con las variantes establecidas en la fase anterior, y relacionadas las distintas áreas con sus correspondientes asociaciones, se procede a su acoplamiento y elaboración de la leyenda, señalando en cada asociación -en orden relativo a su extensión- las diferentes unidades taxonómicas.

\section{APLICACIÓN DE LA PROPUESTA METODOLÓGICA A UN ÁREA PILOTO DE LA PROVINCIA DE ALICANTE}

Como área-piloto, en esta propuesta metodológica, hemos escogido la superficie abarcada por el fotograma $\mathrm{n}^{\circ} 7243 \mathrm{del}$ «vuelo americano» (1956) a E 1/33.000, situada al SW de Alicante y que se integra casi en su totalidad en el espacio comarcal del Bajo Vinalopó.

Morfológicamente, elárea-piloto aparece como un suave glacis cuaternario que desciende desde los $100 \mathrm{~m}$ de la zona de contacto con la alineación montañosa septentrional de la Serra dels Colmenars hasta los 10-20 m del área suroriental, que presenta un notorio proceso de subsidencia ${ }^{13}$.

Litológicamente, se trata de un área con claro predominio cuaternario, donde destaca, con personalidad propia, la presencia de vastas extensiones de superficie de glacis antiguos protegidos por costras calizas (caliche). 
La disposición del relieve y la situación del área comentada definen una característica climática basada en [19]

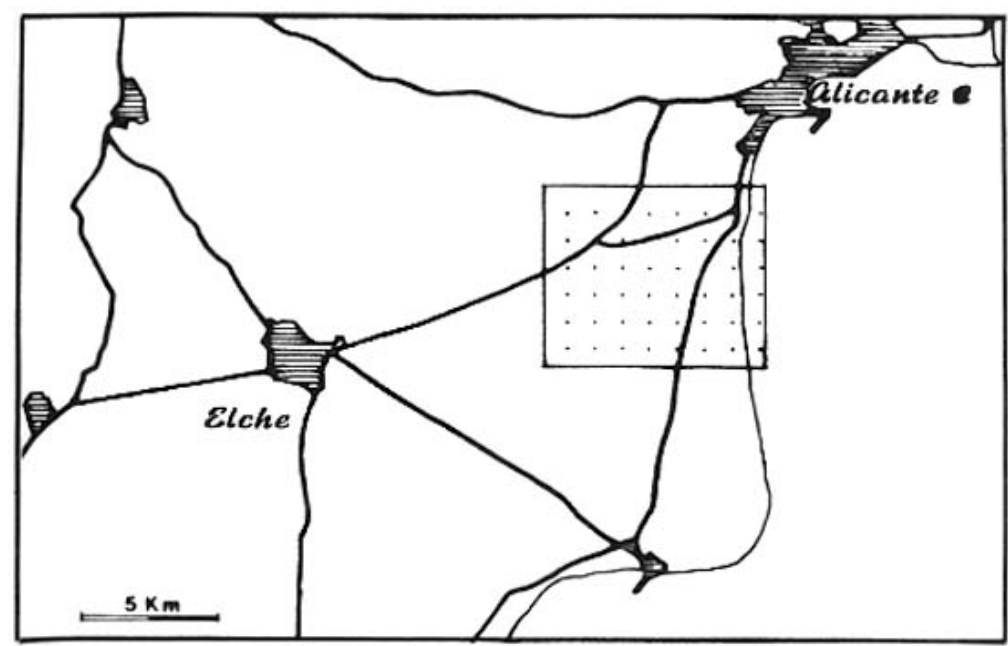

Fig. 1.- Localización del área piloto analizada en el entorno geográfico del Bajo Vinalopó y sur del Campo de Alicante

unas precipitaciones escasas, en torno a los $300 \mathrm{~mm}$. año, y unas temperaturas ligeramente superiores a $\operatorname{los} 18^{\circ} \mathrm{C}$ como media anual; factores, ambos, condicionantes del elevado índice de aridez manifiesto en muchas unidades edáficas comarcales y en el ambiente rexistásico de su rala cobertura vegetal.

Los suelos del sector considerado son resultado, en su mayoría, de la litología del roquedo subyacente y circundante: areniscas calcáreas y calcomargosas del mioceno, así como costras calcáreas que facilitan la aparición de suelos pardo-calizos profundos en complejo con yerma de costra caliza, aparte de los suelos aluvio-coluviales, constituidos sobre sedimentos del cuaternario.

Teniendo en cuenta la combinación de criterios fotográficos directos y factores indirectos, se pueden distinguir en este área un cierto número de unidades (zonas homogéneas); éstas han sido representadas sobre el fotograma por un símbolo complejo constituido por cinco indicadores: tonalidad de la foto (letra mayúscula), litología [20] 


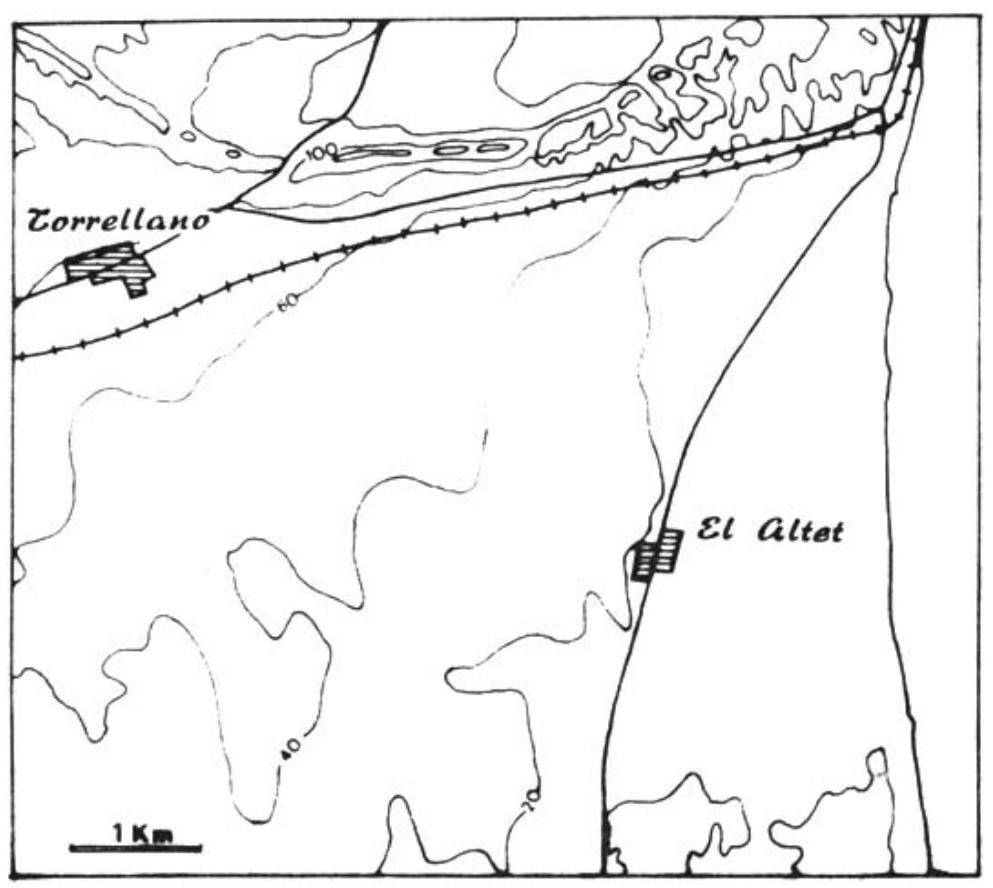

Fig. 2.- Esquema topográfico del área que aparece punteada en la figura 1, correspondiente a la superficie cubierta por el fotograma número 7243 del vuelo de 1956 (vid. fig. 3)

(cifra romana), unidad geomorfológica (letra minúscula), pendiente (subíndices) y ocupación del suelo (letra mayúscula).

La comparación de estas zonas homogéneas con las observaciones del terreno, realizada en la prospección de verificación, junto con la labor de fotointerpretación llevada a cabo, nos ha permitido matizar una serie de conjuntos mayores que corresponden a las asociaciones de suelos que se definen seguidamente ${ }^{14}$ :

\section{Lithic Xerorthents}

\section{Unidades 1 y 8 Símbolos $\mathrm{CIa}_{2} \mathrm{R} / \mathrm{CIa}_{3} \mathrm{R}$}

Typic Xerorthents

El aspecto fotográfico de estas unidades -diferentes tan sólo por el grado de pendiente que presentan- está caracterizado por unas tonalidades grises (C), que, evidentemente, se repiten en otros casos y que son insuficientes para su diferenciación. Los elementos distintivos son de orden fisiográfico, así litológicamente, las areniscas calcáreas y areniscas calcomargosas miocénicas $\operatorname{del}[21]$ 


\title{
CUADRO II \\ RELACIONES ENTRE LOS CRITERIOS FOTOGRÁFICOS Y FISIOGRÁFICOS DE LAS ZONAS HOMOGÉNEAS DEL ÁREA-PILOTO
}

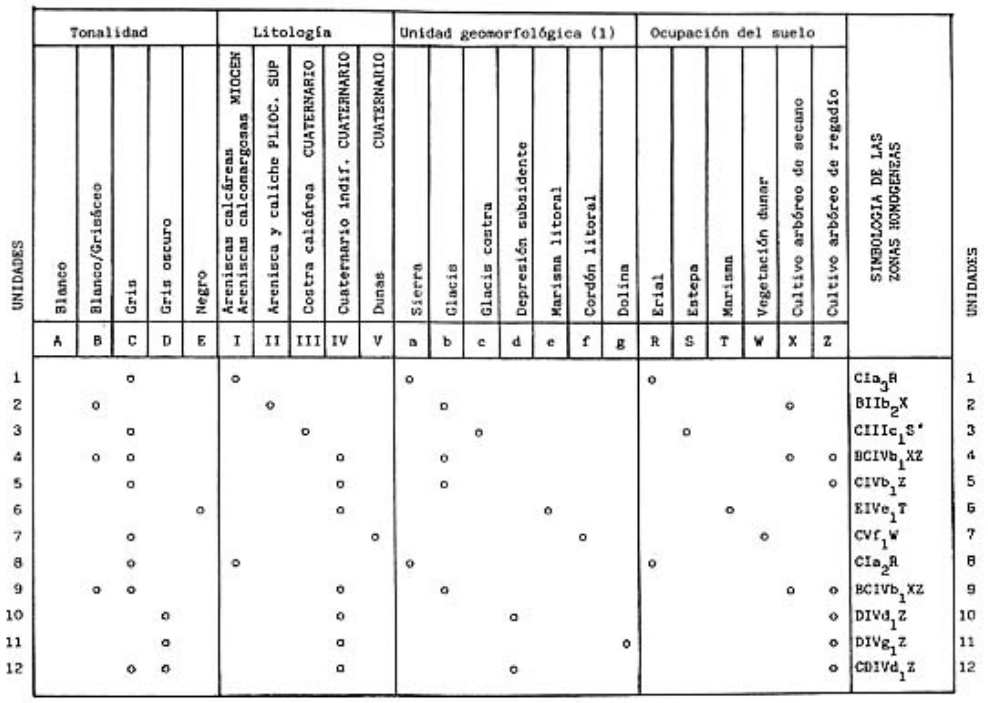

(1) Los subíndices que aparecen en la simbología de las zonas homogéneas hacen referencia al distinto porcentaje de pendiente de las unidades geomorfológicas: 1, de 0 a $2 \% ; 2$, de 2 a $8 \%$; , más de $8 \%$

[22]

sustrato (I) condicionan un paisaje ondulado, de sierra, con pendientes en algunos casos del 2 al $8 \%\left(a_{2}\right)$ y en otros superiores al $8 \%\left(a_{3}\right)$, que explican un nulo aprovechamiento del suelo en una zona típica de erial (R).

Los suelos que integran esta asociación -fundamentalmente, litosuelos y regosuelos- por la topografía ondulada que ocupan y la naturaleza del material geológico sobre el que se originan, son poco evolucionados, de naturaleza pétrea, diaclasados y atacados por meteorización. Se ubican en la Sierra del Colmenar (al N del área señalada) y en su prolongación al W del Collado del Portichuelo.

\section{Typic Xerorthents}

\section{Unidad 2}

\author{
Símbolo $\mathrm{BIIb}_{2} \mathrm{X}$
}

\section{Lithic Xerorthents}

Sobre el fotograma esta unidad da una tonalidad blancogrisácea (B), que la distingue perfectamente de la anterior. Por otro lado, el condicionamiento lítico: areniscas del Plioceno superior (II), y su posición morfológica: glacis meridional del arco montañoso citado $\left(b_{2}\right)$, posibilita no sólo una clara diferenciación en la fase de fotointerpretación, sino, asimismo, una ocupación del suelo tendente al cultivo arbóreo de secano $(\mathrm{X})$, como se pudo comprobar en la prospección de verificación efectuada.

La mayoría de las unidades taxonómicas de esta asociación -regosuelos y litosuelos- que se presentan en laderas de pendientes medias (subíndice 2), están poco evolucionados y ofrecen una pedregosidad variable -visible en algunos casos- por la cercanía a los afloramientos circundantes próximos.

Esta unidad dibuja una mancha -sobre el fotograma- que se dispone alargada de $\mathrm{E}$ a W, sensiblemente paralela a la Sierra del Colmenar. El aprovechamiento se reduce a cultivos 
arbóreos de secano -ausentes en ocasiones (circunstancia que se traduce en el fotograma por unas manchas blanquecinas)- dispuestos en terrazas.

\section{Petrocalcic Xerochrepts}

Typic Xerorthents Unidad $3 \quad$ Símbolo CIIIc,S

\section{Lithic Xerorthents}

[23]

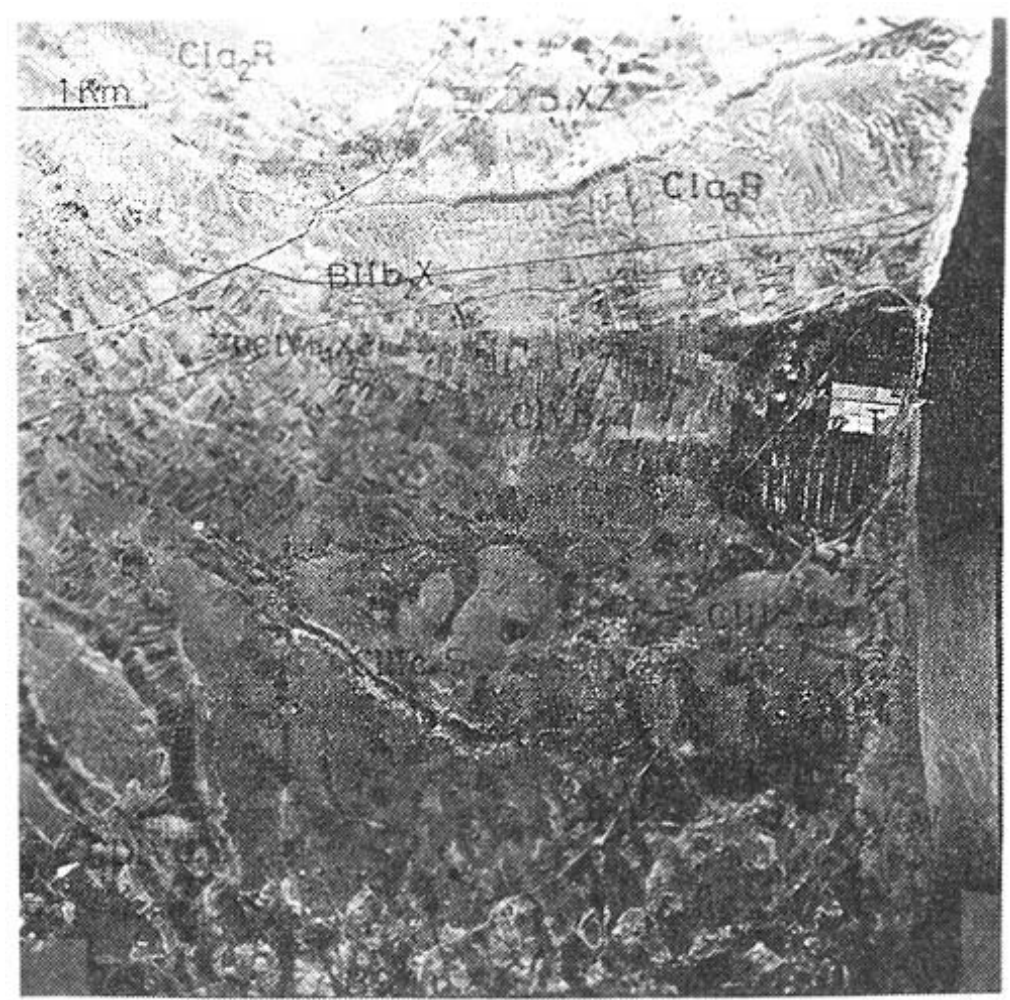

Fig. 3.- Fotograma con delimitación simbólica de las zonas homogéneas

La tonalidad fotográfica que ofrece esta unidad también es gris $(C)$, pero su distinción absoluta viene dada por el aspecto que sus factores líticos y morfológicos proporcionan: se trata de áreas cubiertas por una espesa capa de caliche (III), que matiza una perfecta delimitación sobre el fotograma (tonalidad grisácea punteada), situada sobre un glacis-costra de nula pendiente $\left(b_{1}\right)$, que tan solo posibilita un paisaje estepario, salvo en determinados puntos en los que el menor espesor de la capa caliza y la acción humana han posibilitado el acceso a unos suelos mejores situados por debajo de la costra.

Con todo, si la costra calcárea aflora ${ }^{15}$, circunstancia que sucede en esta unidad con cierta frecuencia, es posible señalar la presencia de unos raquíticos suelos solamente colonizados por algunos líquenes y, en general, una rala cobertura vegetal que matiza un paisaje estepario. Cuando la costra caliza está a algunos centímetros de la superficie, se encuentra cubierta por una capa de suelo pedregoso, pardo o rojizo, calizo, desarrollado sobre sedimentos coluviales relativamente recientes que yacen sobre la costra. [24]

Esta asociación abarca un porcentaje alto de la banda meridional del fotograma analizado: sectores oriental y occidental de El Altet, y zona circundante de los Balsares (49 m). 
IV Typic Xerorthents

Typic Xerofluvents

Unidades 4 y 9

Símbolo BCIVb $\mathrm{XZ}$

Calcixerollic Xerochrepts

Sobre la base de los criterios establecidos -directos e indirectos- ha sido posible distinguir esta asociación que aparece tanto en la unidad número 4 como en la 9 y que se diferencia de los restantes no sólo por la tonalidad de la mancha en el fotograma -blanco grisácea y grissino por su componente lítico: material cuaternario (IV), sobre la superficie del glacis que desciende desde la Sierra del Colmenar en suave pendiente $\left(b_{1}\right)$, circunstancias que contribuyen a una notable utilización agraria $(\mathrm{XZ})$.

Se trata de una asociación de regosuelos, xerorendzinas y suelos pardo calizos (en menor proporción), entes pedológicos que se encuentran asociados a estas topografías de pendientes moderadas y que se desarrollan a partir de sedimentos cuaternarios constituidos por materiales resultantes de la erosión de margas y calizas de las formaciones terciarias aledañas.

\section{Calcixerollic Xerochrepts}

Typic Xerorthents Unidad $5 \quad$ Símbolo $\mathrm{CIVb}_{1} \mathrm{Z}$

Petrocalcic Xerochrepts

Esta unidad se diferencia de la anterior, no sólo por la tonalidad -gris más o menos homogéneo a lo largo de toda la mancha- sino también por las mayores posibilidades de aprovechamiento del suelo (Z), dedicado sobre todo a cultivos de regadío.

Los tipos de suelos que componen esta asociación marcan un predominio de suelos pardo calizos en complejo con regosuelos y algún afloramiento de costra caliza. Se desarrollan a partir de los materiales de un glacis $\left(b_{1}\right)$. Son moderadamente pedregosos, no apreciándose indicios de traslocación de carbonatos en el perfil, a pesar de que, [25] circunstancialmente, pueden presentarse -como hemos indicado- superficies encostradas.

Se localiza en una amplia banda que discurre por el centro del área analizada de W a E, desde el S de Torrellano hasta El Saladar.

\section{Typic Xerorthents}

Aquollic Salorthids

Unidad 6

Símbolo EIVe ${ }_{1} \mathrm{~T}$

Typic Salorthids

Esta unidad ha podido ser delimitada exclusivamente a base de criterios fotográficos directos: la tonalidad negra (E) y textura de la imagen observada no aparecen en el resto del área estudiada. La tonalidad negra está causada por la escasa reflexión de las áreas húmedas. Asimismo, observaciones complementarias permiten distinguir la cuadrícula de antiguas salinas -actualmente abandonadas- que caracterizan a esta zona de marisma (T).

Los suelos que forman esta asociación -salinos, por su ubicación próxima al litoral y por su morfología- se desarrollan 


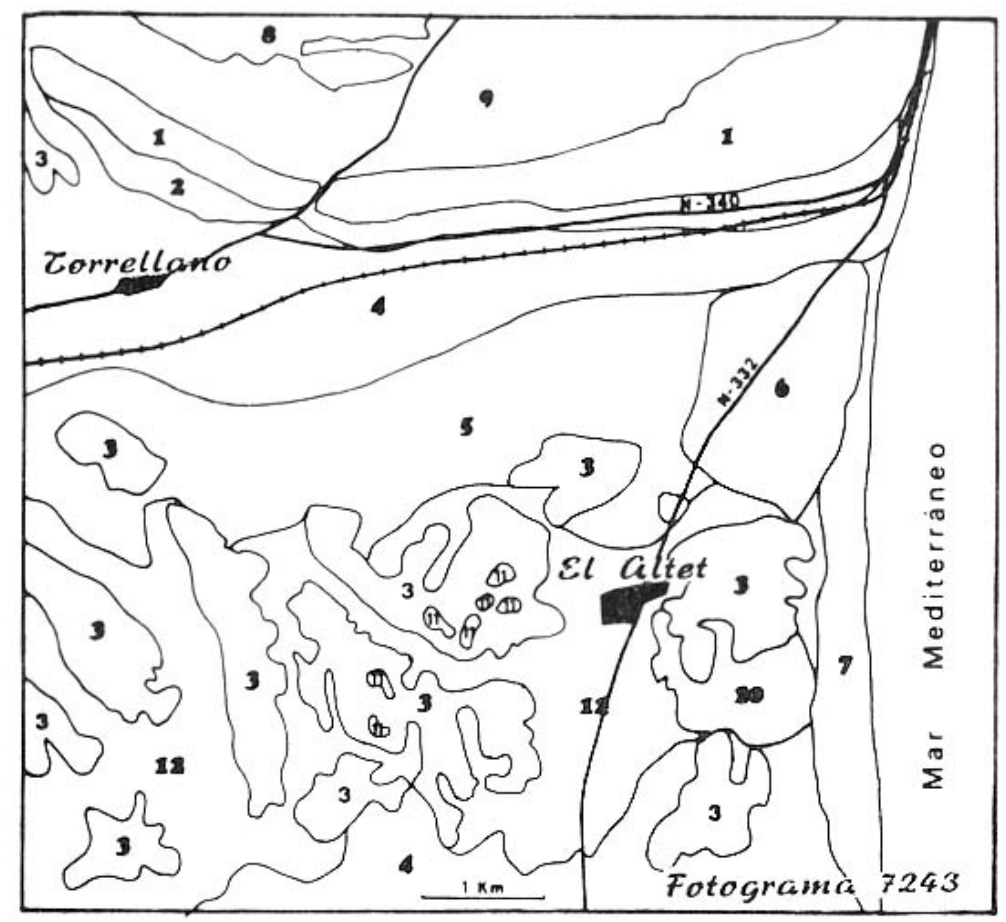

[26]

Fig. 4.- Delimitación de las asociaciones cartográficas de suelos de la zona de estudio

a partir de sedimentos cuaternarios caracterizados por un depósito masivo arcillo-limoso. Se localizan al E del fotograma, área de El Saladar (cercanías de Urbanova), marisma de topografía llana. Se trata de suelos jóvenes, sin desarrollo de horizontes edáficos, con nivel freático próximo a la superficie y de alta salinidad ${ }^{16}$. Su aprovechamiento agrícola es nulo, dado que los condicionamientos edáficos sólo permiten la existencia de una vegetación halófila.

\section{Typic Xeropsamment}
Aquic Xerorthents
Unidad 7
Símbolo $\mathrm{CVf}_{1} \mathrm{~W}$

El elemento distintivo de esta unidad radica en la tonalidad gris de la mancha (C), así como la textura punteada que ofrece, aparte de ciertas consideraciones de índole fisiográfica que, sin duda, matizan su individualización: morfológicamente se estructura como un cordón de dunas litorales (f), con una vegetación psamófila que ha sufrido una fuerte regresión como consecuencia de la acción antrópica (urbanizaciones, complejos residenciales).

Los suelos arenosos que constituyen esta asociación son poco evolucionados, presentan unas texturas gruesas -dado su desarrollo sobre arenales costeros ${ }^{17}$. Se trata de regosuelos que pueden adscribirse -en algún caso- al tipo de yerma de arenas de la clasificación de Kubiena. Se sitúan a lo largo de la franja costera oriental del área-piloto, integrando las playas de Urbanova y de los Arenales del Sol.

\section{Calcixerollic Xerochrepts}

Typic Xerorthents

Unidades 10-11-12

Símbolos DIVd $\mathrm{Z}$

Typic Xerofluvents

$\mathrm{CDVd}_{1} \mathrm{Z}$ 
Estas unidades se diferencian en el fotograma por unas tonalidades gris-gris oscuro (CD). El factor indirecto, de tipo fisiográfico, que mejor las caracteriza es el formar parte, bien de áreas deprimidas (depresión de El Altet-Fondo de la Senieta), o de amplios canales (canyades) ${ }^{18}$ de orientación NW-SE que cruzan las formaciones de caliche a 3 ó $4 \mathrm{~m}$ de desnivel, o incluso, conformando pequeños enclaves cerrados, a modo de dolinas que, en [27]

\section{CUADRO III \\ RELACIÓN ENTRE LAS ASOCIACIONES CARTOGRÁFICAS DE SUELOS Y LAS UNIDADES FOTOINTERPRETADAS EN EL FOTOGRAMA 7243}

\begin{tabular}{|c|c|c|c|c|c|}
\hline Asociacion & \multicolumn{2}{|c|}{ Tipos de suelos } & Unidades & Sfebolos & Unidad de paisaje \\
\hline I & $\begin{array}{l}\text { LITHIC XERORTHENTS } \\
\text { TYPIC XERORTHENTS }\end{array}$ & $\begin{array}{l}\text { Litosuelos } \\
\text { Regosuelos }\end{array}$ & $1-8$ & $\mathrm{Cra}_{3} \mathrm{R} / \mathrm{Cra}_{2} \mathrm{R}$ & Areas montaflosas \\
\hline II & $\begin{array}{l}\text { TYPIC XERORTHENTS } \\
\text { LITHIC XERORTHENTS }\end{array}$ & $\begin{array}{l}\text { Regosuelos } \\
\text { Litosuelos }\end{array}$ & 2 & $\mathrm{BIIb}_{2} \mathrm{X}$ & $\begin{array}{l}\text { Piedemonte de las } \\
\text { areas montañosas }\end{array}$ \\
\hline III & $\begin{array}{l}\text { PETROCALCIC XEROCHREPTS } \\
\text { TYPIC XERORTHENTS } \\
\text { LITHIC XERORTHENTS }\end{array}$ & $\begin{array}{l}\text { Costra caliza } \\
\text { Regosuelos } \\
\text { Litosuelos }\end{array}$ & 3 & $\mathrm{CrIIc}_{1} \mathrm{~s}$ & $\begin{array}{l}\text { Fornaciones de costra } \\
\text { caliza }\end{array}$ \\
\hline IV & $\begin{array}{l}\text { TYPIC XERORTHENTS } \\
\text { TYPIC XEROFLUVENTS } \\
\text { CALCIXEROLLIC XEROCHREPTS }\end{array}$ & $\begin{array}{l}\text { Regosuelos } \\
\text { Xerorendzinas } \\
\text { Pardo calizo }\end{array}$ & $4-9$ & $\mathrm{BCrVb}_{1} \mathrm{xz}$ & $\begin{array}{l}\text { Cultivos arboreos so- } \\
\text { bre glacis }\end{array}$ \\
\hline $\mathrm{v}$ & $\begin{array}{l}\text { CALCIXEROLLIC XEROCHREPTS } \\
\text { TYPIC XERORTHENTS } \\
\text { PETROCALCIC XEROCHREPTS }\end{array}$ & $\begin{array}{l}\text { Pardo calizo } \\
\text { Regosuelos } \\
\text { Costra caliza }\end{array}$ & 5 & $\mathrm{crvb}_{1} 2$ & $\begin{array}{l}\text { Cultivos hortorrutico } \\
\text { las sobre glacis }\end{array}$ \\
\hline $\mathrm{vI}$ & $\begin{array}{l}\text { TYPIC XERORTHENTS } \\
\text { AQUOLLIC SALORTHIDS }\end{array}$ & $\begin{array}{l}\text { Regosuelos } \\
\text { Suelos salínos }\end{array}$ & 6 & $\operatorname{ErVe}_{1} \mathrm{~T}$ & Marisma - Saladar \\
\hline VII & $\begin{array}{l}\text { TYPIC XEROPSANQEENT } \\
\text { AQUIC XERORTHENTS }\end{array}$ & Suelos arenosos & 7 & evr $r_{1}$ & $\begin{array}{l}\text { Cordon de arenas } \\
\text { Dunas litorales }\end{array}$ \\
\hline vinI & $\begin{array}{l}\text { CALCIXEROLLIC XEROCHREPTS } \\
\text { TYPIC XERORTHENTS } \\
\text { TYPIC XEROFLUVENTS }\end{array}$ & $\begin{array}{l}\text { Pardo calizo } \\
\text { Regosuelos } \\
\text { Xerorendzinas }\end{array}$ & $10-11-12$ & $\operatorname{DIVd}_{1} z / \operatorname{COIVd}_{2} z$ & $\begin{array}{l}\text { Areas depriaidas, can- } \\
\text { yades y dolinas con al } \\
\text { to aprovechaniento agr } \underline{\underline{1}} \\
\text { cola }\end{array}$ \\
\hline
\end{tabular}

[28]

algún caso funcionan con su propio sumidero y ofrecen los clásicos manchones de terra rossa.

Los suelos que componen esta asociación -suelos pardo calizos profundos en su mayoríase desarrollan en depresiones rellenas por materiales blandos: margas y arcillas terciarias y material cuaternario. Son suelos moderadamente pedregosos, ofrecen un epipedon ochrico con un límite inferior brusco, cuando reposa directamente sobre el material subyacente, o bien gradual, cuando pasa a un horizonte cámbico. No se aprecian indicios de traslocación de carbonatos en el perfil, a pesar de que, ocasionalmente, puedan presentarse superficies encostradas. Se trata de áreas con aprovechamiento agrícola bueno.

[29]

\section{CITAS BIBLIOGRÁFICAS}

ALEIXANDRE, T. y PINILLA, A., «Mineralogía de unos suelos mediterráneos de las provincias de Almería, Murcia y Alicante», Anales de Edafología y Agrobiología, 1968.

BUOL, S. W. and oth., Soil genesis and classification, Iowa St. Univ. Press, Ames, 1973.

BURINGH, P., Application of aeriel photographs in soil surveys. Manual of photographic Interpretation, Washington, 1960.

CANALES SELVA, S., Génesis y clasificación de los suelos en la zona baja del valle del Segura, Tesis doctoral, 1972.

CARPENA, O. y otros, Estudio agrobiológico y aspectos económicos de los partidos de Orihuela y Dolores (Alicante), CSIC, 1969. 
CARPENA, O. y otros, «Áreas de suelo natural en la Vega Baja del Segura. Formación de suelos arenosos en el término de San Fulgencio», Anales de Edafología y Agrobiología, 1972.

C.E.B.A.C., Estudio agrobiológico de la provincia de Sevilla, CSIC, Sevilla, 1962.

C.E.B.A.C., Estudio agrobiológico de la provincia de Cádiz, CSIC, Sevilla, 1963.

C.E.B.A.C., Estudio agrobiológico de la provincia de Córdoba, CSIC, Sevilla, 1972.

C.E.B.A.S., Mapa de suelos de la provincia de Cáceres, CSIC, Salamanca, 1972.

DE LA ROSA, D., «Perspectivas actuales de la cartografía y evaluación de los suelos», Anales de Edafología y Agrobiología, Madrid, 1981.

DEL VILLAR, E. H., Los suelos de la península Luso-Ibérica, Madrid, 1937.

FERNÁNDEZ CALDAS, E. y otros, Suelos de regiones volcánicas. Tenerife, Col. Viera y Clavijo, Secretariado de Publicaciones de la Universidad de La Laguna, Sta. Cruz de Tenerife, 1982, $250 \mathrm{pp}$.

GOOSEN, D., Interpretación de fotos aéreas y su importancia en los levantamientos de suelos, FAO, Roma, 1968. [30]

GUMUZZIO, J.-MATARREDONA COLL, E., Formaciones edáficas del Campo de Alicante, Universidad de Alicante, Instituto Universitario de Geografía, Alicante, 1983.

INSTITUTO NACIONAL DE EDAFOLOGÍA Y AGROBIOLOGÍA, Mapa de suelos de España. E. 1/1.000.000. Descripción de las asociaciones y tipos principales de suelos, CSIC, Madrid, 1968.

I.N.I.A., Mapas provinciales de suelos. Cádiz. E. 11200.000, Ministerio de Agric., Madrid, 1971.

I.N.I.A., Mapas provinciales de suelos. Sevilla. E. 1/200.000, Ministerio de Agric., Madrid, 1975.

ÍÑIGUEZ, J. y otros, «Mapa de suelos de Navarra. E. 1/50.000. Hoja 141. Pamplona», Príncipe de Viana, Año 2, nº 2, Diputación Foral, Pamplona, 1982.

JENNY, H., Factors of soil formation, Mc Graw Hill, New York, 1941.

JIMÉNEZ, R.-GUERRA, A., «Inceptisoles sobre materiales carbonatatos en clima mediterráneo de la provincia de Castellón de la Plana, Anales de Edafología y Agrobiología, Madrid, 1980.

JIMÉNEZ, R., «Estudio de algunas formaciones edáficas características, desarrolladas bajo el sector semiárido de la provincia de Castellón de la Plana», Actas de la III Reunión Nacional del Grupo Español de Trabajo del Cuaternario, 1977.

LAOMIRANT, H., Photographies aeriennes et Geologie, Tervuren, 1972.

LOOTENS DE MUYNCK, M. T. VERHEYE, W., «Photointerpretation et cartographie morphopedologique en pays mediterraneen (Argolis, Grece)», Mediterranée, nº 4, 1976.

LÓPEZ VERGARA, M. L., Manual de fotogeología, J. E. N., Madrid, 1971.

MATARREDONA COLL, E., Estudio Geográfico del Alto Vinalopó, I. E. A., Alicante, 1983, 370 pp.

MATARREDONA COLL, E., Asociaciones cartográficas de suelos del Bajo Vinalopó, en prensa.

MINISTERIO DE AGRICULTURA, Los grupos principales de suelos de la España peninsular. E. 1/1.300.000, Mapa Agronómico Nacional, Madrid, 1958.

PIQUERAS, J., «El estudio de los suelos en el País Valenciano», Cuadernos de Geografía, n² 27, Valencia, 1980.

POWELL, J. C. SPRINGER, M. E., Composition and precision of classification of several mapping units of the Appling, Cecil and Lloyd series in Walton County, Georgia, 1965.

SÁNCHEZ, J. A., «Formaciones geodáficas en las terrazas cuaternarias litorales de las provincias de Alicante y Murcia», Actas de la I Reunión Nacional del Grupo de Trabajo del Cuaternario, Madrid, 1973. 
SÁNCHEZ, J. A., «El cuaternario de la región semiárida suroriental de España: formaciones edafológicas en el Cabo de las Huertas», Actas de la III Reunión Nacional del Grupo Español de Trabajo del Cuaternario, Madrid, 1977.

SIMONSON, R. W., Lessons from the first half century of soil survey. II. Mapping of soils, Soil Scient. 74, 1952.

WILDEING, L. P. and ot., Variation of soil morphological properties within Miami, Celina and Crosby mapping units in West-central Ohio, Soil Sci. Soc. Am. Proc., 29, 1965.

\section{BIBLIOGRAFÍA}

1 DE LA ROSA, D., «Perspectivas actuales de la cartografía y evaluación de los suelos», Anales de Edafología y Agrobiología, t. XV, núms. 11-12, Madrid, 1981, p. 2.293.

2 DE LA ROSA, D., op. cit., p. 2.309.

3 DE LA ROSA, D., op. cit., p. 2.309.

4 GUERRA DELGADO, A., Mapa de suelos de España. E 1/1.000.000, CSIC, Instituto Nacional de Edafología y Agrobiología «José M. a Albareda», Madrid, 1968, 119 pp. + 1 mapa.

5 Además podemos incluir: ÍÑIGUEZ, J. y otros. «Mapa de suelos de Navarra. E 1/50.000. Hoja 141. Pamplona», Príncipe de Viana, Diputación Foral Navarra, Servicio de Publicaciones de la Institución «Príncipe de Viana», Año 2, núm. 2, Pamplona, 1982, pp. 187-222. FERNÁNDEZ CALDAS, E. y otros, Suelos de regiones volcánicas. Tenerife, Col. Viera y Clavijo, Secretariado de Publicaciones de la Universidad de La Laguna, Sta. Cruz de Tenerife, 1982, 250 pp. + 1 mapa.

6 PIQUERAS HABA, J., «El estudio de suelos en el País Valenciano. Estado actual», Cuadernos de Geografía, núm. 27, Valencia, 1980, p. 221.

7 Interesa destacar el estudio sobre suelos del municipio de Denia, de Sánchez; el trabajo de Pujol sobre los suelos del Camp de Morvedre; o la tesis doctoral de Antolín sobre formaciones edáficas de la comarca de la Costera. Vid.: PIQUERAS HABA, J., op. cit., p. 221.

8 MATARREDONA, E., Estudio Geográfico del Alto Vinalopó, I.E.A., Alicante, 1983, pp. 60-72.

9 GUMUZZIO, J. y MATARREDONA, E., Formaciones edáficas del Campo de Alicante, Universidad de Alicante, Alicante, 1983, 51 pp.

10 MATARREDONA, E., Asociaciones cartográficas de suelos del Bajo Vinalopó, en prensa.

11 LAOMIRANT, H., Photographies aeriennes et Geologie, Tervuren, 1972.

BURINGH, P., Application of aerial photographs in soil surveys. Manual of photographic Interpretation, Washington, 1960. GOOSEN, D., Interpretación de fotos aéreas y su importancia en el levantamiento de suelos, FAO, Roma, 1968.

12 LOOTENS DE MUYNCK, M. T. et VERHEYE, W., «Photointerpretation et cartographie morphopedologique en pays mediterraneen (Argolis, Grece)», Mediterranée, $\mathrm{n}^{\circ}$ 4, 1976, pp. 39-51.

13 GOZÁLVEZ PÉREZ, V., El Bajo Vinalopó. Geografía Agraria, Valencia, 1977, p. 12.

14 Las letras y números que aparecen entre paréntesis en el texto explicativo de las distintas asociaciones hacen referencia a la simbología utilizada en la caracterización de cada zona homogénea, que queda reflejada en el Cuadro II.

15 En las proximidades del K-11 de la N-332, al lado mismo de la carretera, se percibe -en una cornisa abarrancada- una capa de costra de más de $50 \mathrm{~cm}$. de espesor y bajo ella una serie de estratos calizos y margosos que cubren un último horizonte de limos rojos y blanquecinos situados a 1,40 m. del nivel de superficie. Vid.: 
MATARREDONA COLL, E., op. cit., en prensa.

16 El análisis granulométrico de un perfil en el área de El Saladar refleja unos horizontes de clase textural franco-limosa (20,9\% de arenas, $75,3 \%$ de limos y 3,8 \% de arcillas), con unos pH que se sitúan en torno a 8 . Vid.: MATARREDONA, E., op, cit., en prensa.

17 La granulometría del horizonte medio de un perfil analizado entre Urbanova y los Arenales del Sol permite calibrarlo de arenoso-franco ( $80,9 \%$ de arenas, $17,9 \%$ de limos y 1,2 de arcillas). Vid.: MATARREDONA, E., op. cit., en prensa.

18 GOZÁLVEZ PÉREZ, V., op cit., p. 17. 